\title{
Identification of FOXM1 as a specific marker for triple-negative breast cancer
}

\author{
YANLI TAN $^{1-3}$, QIXUE WANG $^{4,5}$, YINGBIN XIE ${ }^{6}$, XIAOXIA QIAO $^{6}$, \\ SHUN ZHANG ${ }^{3}$, YANAN WANG ${ }^{3}$, YONGBIN YANG ${ }^{2}$ and BO ZHANG ${ }^{1}$
}

${ }^{1}$ Department of Pathology, School of Basic Medical Sciences, Peking University Health Science Center, Beijing 100191; ${ }^{2}$ Department of Pathology, Hebei University Medical College; ${ }^{3}$ Department of Pathology, Affiliated Hospital of Hebei University, Baoding, Hebei 071000; ${ }^{4}$ Department of Neurosurgery; ${ }^{5}$ Laboratory of Neuro-Oncology, Tianjin Neurological Institute, Tianjin 300052; ${ }^{6}$ Department of Neurosurgery, Affiliated Hospital of Hebei University, Baoding, Hebei 071000, P.R. China

Received June 7, 2018; Accepted August 30, 2018

DOI: 10.3892/ijo.2018.4598

\begin{abstract}
The present study aimed to identify the therapeutic role of the forkhead box M1 (FOXM1)-associated pathway in triple-negative breast cancer (TNBC). Using a Cancer Landscapes-based analysis, a gene regulatory network model was constructed. The present results demonstrated that FOXM1 occupies a key position in gene networks and is a critical regulatory gene in breast cancer. Using breast carcinoma gene expression data from The Cancer Genome Atlas, it was identified that FOXM1 expression was increased in the basal-like breast cancer subtype compared with other breast cancer subtypes. RNA-sequencing analysis of MDA-MB-231 cells treated with 4 and $10 \mu \mathrm{l} / \mathrm{ml}$ Thiostrepton identified 662 and 5,888 significantly differentially expressed genes, respectively. The Kyoto Encyclopedia of Genes and Genomes pathway enrichment analyses demonstrated that FOXM1 was highly associated with multiple biological processes and was markedly associated with metabolic pathways in TNBC. The use of Search Tool for the Retrieval of Interacting Genes/Proteins provided a critical assessment and integration of protein-protein interactions, and demonstrated the multiple important functions of FOXM1 in TNBC. Real-time cell analysis, reverse transcription-quantitative polymerase chain reaction and immunofluorescence staining were used to assess the anti-tumor activity of Thiostrepton in TNBC cells in vitro. The present results identified that suppression of FOXM1 using Thiostrepton inhibited MDA-MB-231 cell proliferation and the expression of cell cycle-associated genes, including cyclin A2, cyclin B2, checkpoint kinase 1, centrosomal
\end{abstract}

Correspondence to: Professor Bo Zhang, Department of Pathology, School of Basic Medical Sciences, Peking University Health Science Center, 38 Xueyuan Road, Beijing 100191, P.R. China

E-mail: zhangbo@bjmu.edu.cn

Key words: forkhead box M1, breast cancer, triple-negative breast cancer, molecular, subtype protein 55 and polo like kinase 1. Immunofluorescence staining analysis demonstrated that vimentin, filamentous actin and zinc finger E-box-binding homeobox 1 were all decreased following treatment with Thiostrepton. Furthermore, a BALB/C nude mouse subcutaneous xenograft model was used to verify the function of FOXM1 in vivo. The present results demonstrated that FOXM1 inhibition significantly suppressed MDA-MB-231 cell tumorigenesis in vivo. Overall, the present results suggested that FOXM1 is a key gene that serves important roles in multiple biological processes in TNBC and that it may serve as a novel therapeutic target in TNBC.

\section{Introduction}

Breast cancer is the most common invasive cancer and is a leading cause of cancer-associated mortality in women $(1,2)$. Breast cancer is a heterogeneous and complex disease that may be classified into different subtypes based on the estrogen receptor (ER), progesterone receptor (PR) and human epidermal growth factor receptor 2 (HER2) expression status. Triple-negative breast cancer (TNBC) is an aggressive subtype of breast cancer characterized by a lack of ER, PR and HER2 expression (3). TNBC is associated with early metastasis, high grade disease, a high tumor proliferation rate, drug resistance and poor prognosis $(4,5)$. Due to a lack of molecular targets for drugs, TNBC therapies are restricted to conventionally available chemotherapies, including endocrine therapies and molecular targeted treatments (6). Patients with TNBC are not able to benefit from the currently available targeted therapies, including hormone therapies and HER2-based therapies (7). Therefore, effective therapeutic approaches for TNBC are urgently required. There is additionally a critical requirement to identify the responsible genes of TNBC and to identify novel therapeutic strategies for patients with TNBC $(8,9)$.

Forkhead box M1 (FOXM1) is a member of the fork head/winged-helix family of proteins, which are involved in numerous biological processes, including cell differentiation, cell proliferation, cell cycle progression, DNA damage repair, tissue homeostasis, angiogenesis and apoptosis (10). FOXM1 is overexpressed in various human malignancies, 
including breast cancer (11). A complete understanding of the regulation and role of FOXM1 in cancer may demonstrate its application as a biomarker for cancer diagnosis and a target for treatment (12). The expression of FOXM1 in breast cancer molecular subtypes and its interaction with associated genes have been described in recent studies; eukaryotic elongation factor 2 kinase and integrin $\beta 1$ have been determined to be involved in the FOXM1 network (13-15). However, the therapeutic role of the FOXM1-associated pathway in TNBC remains unclear.

In the present study, the role of FOXM1 in TNBC was examined using bioinformatics analysis, including the Cancer Landscapes online tool, the Search Tool for the Retrieval of Interacting Genes/Proteins (STRING) database and Database for Annotation, Visualisation and Integrated Discovery (DAVID). The present results were additionally verified by in vitro and in vivo experiments. Furthermore, it was demonstrated that a FOXM1 inhibitor significantly suppressed the growth of MDA-MB-231 breast cancer cells in vitro and in vivo. These results provide a better understanding of the regulatory mechanisms of FOXM1 and suggest that FOXM1 may be a novel molecular therapeutic target for TNBC.

\section{Materials and methods}

Drugs and treatments. Thiostrepton, a specific inhibitor of FOXM1, was purchased from Abcam (Cambridge, UK; cat. no. ab143458) and was dissolved in dimethyl sulfoxide (DMSO; Sigma-Aldrich; Merck KGaA, Darmstadt, Germany) to generate a $50 \mu \mathrm{M}$ stock solution and stored at $-20^{\circ} \mathrm{C}$. For all working doses, the stock solution of Thiostrepton was diluted with culture medium. To suppress MDA-MB-231 cell growth in vitro, the cells were treated with 4 and $10 \mu \mathrm{M}$ Thiostrepton for $48 \mathrm{~h}$. The negative control group was treated with an equal volume of DMSO.

Antibodies. Anti-polo like kinase 1 (PLK1) and anti-zinc finger E-box-binding homeobox 1 (ZEB1) antibodies were obtained from Boster Biological Technology (Pleasanton, CA, USA; cat. no. P00182-1) and Santa Cruz Biotechnology, Inc. (Dallas, TX, USA; H102; cat. no. sc-25388), respectively. Anti-cyclin-dependent kinase 1 (CDK1; cat. no. 9116), anti-G2/mitotic-specific cyclin-B1 (CCNB1; cat. no. 4138) were purchased from Cell Signaling Technology, Inc. Anti-filamentous actin (F-actin) antibodies were purchased from Abcam(Cambridge,UK,cat.no.ab205).Anti-proliferation marker protein Ki-67 (Ki-67; cat. no. ZA-0502) and anti-vimentin antibodies (cat. no. ZA-0511), in addition to biotin-conjugated goat anti-rabbit immunoglobulin $\mathrm{G}(\mathrm{IgG})$ secondary antibody (cat. no. TA130016) were obtained from OriGene Technologies, Inc. (Beijing, China).

Cell lines and cell culture. The MDA-MB-231 cell line was purchased from the American Type Culture Collection (Manassas, VA, USA). The cells were maintained in RPMI-1640 medium (Gibco; Thermo Fisher Scientific, Inc., Waltham, MA, USA) supplemented with $10 \%$ fetal bovine serum (Gibco; Thermo Fisher Scientific, Inc.), $100 \mathrm{U} / \mathrm{ml}$ penicillin and $100 \mu \mathrm{g} / \mathrm{ml}$ streptomycin (Sigma-Aldrich; Merck
$\mathrm{KGaA}$ ) and were cultured in a humidified incubator at $37^{\circ} \mathrm{C}$ with $5 \% \mathrm{CO}_{2}$.

Reverse transcription-quantitative polymerase chain reaction $(R T-q P C R)$. Total RNA was extracted from MDA-MB-231 cells using TRIzol ${ }^{\circledR}$ reagent (Invitrogen; Thermo Fisher Scientific, Inc.) according to the manufacturer's protocol. For RT-qPCR, the Qiagen One Step RT-PCR kit (Qiagen GmbH, Heidelberg, Germany; cat. no. 210212) was used. Subsequently, $2 \mu \mathrm{g}$ total RNA was used as the template for RTqi. cDNA was synthesized from total RNA according to the following steps: One cycle at $42^{\circ} \mathrm{C}$ for $1 \mathrm{~h}$ and $70^{\circ} \mathrm{C}$ for $15 \mathrm{~min}$. RT-qPCR was performed using SYBR-Green Master Mix (Applied Biosystems; Thermo Fisher Scientific, Inc.). Reactions for RT-qPCR assays were run in a CFX96 ${ }^{\mathrm{TM}}$ PCR cycler (Bio-Rad Laboratores, Inc., Hercules, CA, USA) according to the following steps: 5 min preheating and denaturation at $95^{\circ} \mathrm{C}$; 40 cycles at $95^{\circ} \mathrm{C}$ for $15 \mathrm{sec} ; 53-58^{\circ} \mathrm{C}$ for $1 \mathrm{~min}$; and a final extension step at $72^{\circ} \mathrm{C}$ for $10 \mathrm{~min}$. GAPDH was used to normalize the relative $\mathrm{mRNA}$ expression levels. The specificity of the RT-qPCR primers was confirmed using melt curve analysis and electrophoresis on $1.2 \%$ agarose gels stained with GelRed fluorescent dye (Biotium, Inc., Freemont, CA, USA). Data analysis was performed using the $2^{-\Delta \Delta \mathrm{Cq}}$ method (16). The primer sequences were as follows: FOXM1 forward, 5'-CCTT CTGGACCATTCACCCC-3' and reverse, 5'-TCACCGGGAA CTGGATAGGT-3'; cyclin B2 (CCNB2) forward, 5'-AGT TCCAGTTCAACCCACCAA-3' and reverse, 5'-TTGCAG AGCAAGGCATCAGA-3'; cyclin A2 (CCNA2 forward, 5'-CT CTACACAGTCACGGGACAAAG-3' and reverse, 5'-CTG TGGTGCTTTGAGGTAGGTC-3'; centrosomal protein 55 (CEP55) forward, 5'-TCGACCGTCAACATGTGC AGCA-3' and reverse, 5'-GGCTCTGTGATGGCAAACTCATG-3'; checkpoint kinase 1 (CHEK1) forward, 5'-ATCAACTCATG GCAGGGGTG-3' and reverse, 5'-TCCAGCGAGCATTGC AGTAA-3'; PLK1 forward, 5'-AGCCCCTCACAGTCCTCA ATA-3' and reverse, 5'-TGTCCGAATAGTCCACCCAC-3'; GAPDH forward, 5'-GGTGGTCTCCTCTGACTTCAACA-3' and reverse, 5'-GTTGCTGTAGCCAAATTCGTTGT-3'.

Immunohistochemistry. Tumor tissues were fixed with $10 \%$ formaldehyde for $24 \mathrm{~h}$ at room temperature, embedded in paraffin and cut into $4 \mu \mathrm{m}$ thick sections. For immunohistochemistry, the primary Ki-67 antibody (1:100) was used according to the manufacturer's protocol. Each section was blocked with $3 \%$ hydrogen peroxide for $10 \mathrm{~min}$ at $37^{\circ} \mathrm{C}$. The sections were incubated with the primary antibody overnight at $4^{\circ} \mathrm{C}$. Subsequently, the sections were incubated with biotin-conjugated goat anti-rabbit $\mathrm{IgG}$ secondary antibodies at a dilution of 1:100 for $20 \mathrm{~min}$ at room temperature. The staining was observed using a Nikon microscope (Nikon Corporation, Tokyo, Japan; magnification, $\mathrm{x} 40$ )

Immunofluorescence staining. After $48 \mathrm{~h}$ of treatment with Thiostrepton, MDA-MB-231 cells were washed with PBS and fixed in $4 \%$ paraformaldehyde for $30 \mathrm{~min}$ at room temperature. PBS with Tween-20 and 5\% bovine serum albumin (Cell Signaling Technology, Inc., Danvers, MA, USA) was used to block the washed cells for $30 \mathrm{~min}$ at $37^{\circ} \mathrm{C}$. The cells were 
incubated with primary antibodies at $4^{\circ} \mathrm{C}$ overnight and were subsequently stained with a fluorescent secondary antibody (Alexa-Fluor $^{\mathrm{TM}} 594$ donkey anti-rabbit IgG; 1:1,000; cat. no. A-21207; Invitrogen; Thermo Fisher Scientific, Inc.) at $37^{\circ} \mathrm{C}$ for $30 \mathrm{~min}$ in the dark. DAPI (cat. no. C1006; Beyotime Institute of Biotechnology, Haimen, China) was used to stain the nucleus for $15 \mathrm{~min}$ at room temperature. Immunopositive cells were observed under a fluorescence microscope. The sections were observed under a Nikon ECLIPSE 80i fluorescent microscope (Nikon Corporation; magnification, $\mathrm{x} 40$ ) and the images were captured using a Nikon digital camera DXM1200 (Nikon Corporation).

Real-time cell proliferation assay (RTCA). Cell proliferation was determined by an RTCA assay. In total, 3,000 cells (100 $\mu \mathrm{l} /$ well) were seeded into an E-Plate. After a 24-h incubation, the cells were treated with $0.5,1,2,5$, 10, 20 and $40 \mu \mathrm{M}$ Thiostrepton for $72 \mathrm{~h}$ at $37^{\circ} \mathrm{C}$. Cell proliferation was automatically monitored in each well using the xCELLigence system (ACEA Biosciences, Inc., San Diego, CA, USA) and was expressed as the Cell Index (CI). $\mathrm{CI}$ is regarded as an indicator of cell proliferation. The $\mathrm{CI}$ values were automatically calculated using RTCA software (version 2.0; ACEA Biosciences, Inc.) and were recorded every $15 \mathrm{~min}$ for $96 \mathrm{~h}$. Data analysis was performed using RTCA software.

Tumor xenograft growth in nude mice. In total, 20 BALB/C nude mice (female; 5-6 weeks of age; weighing 20-25 g) were purchased from the Chinese Academy of Medical Science Cancer Institute (Beijing, China). The mice were housed in a specific-pathogen-free grade animal center at standard temperature $\left(23 \pm 1^{\circ} \mathrm{C}\right)$ and humidity $(45-55 \%)$ conditions, and a standard 12-h dark/12-h light cycle. The mice were given chow and water ad libitum. Tumor xenografts were generated via the subcutaneous injection of $2 \times 10^{6}$ MDA-MB-231 cells into the right front leg of the mice ( $n=8$ mice/group). Tumor xenografts were classified into two groups: The negative control (NC) group or the treatment with Thiostrepton (50 mg/kg, every other day) group. Tumor sizes were measured every other day using micrometer calipers, and tumor volumes were calculated according to the following formula: Tumor volume $\left(\mathrm{mm}^{3}\right)=0.5 \times \mathrm{D} \mathrm{x} \mathrm{d}$, where $\mathrm{d}$ and D represent the shortest and the longest diameters, respectively. It was possible to measure the tumor sizes with a caliper due to the subcutaneous location of the tumors. All tumors were measured by one investigator to prevent observational differences. Day 35 was selected as the humane endpoint to terminate the present study, based on the tumor size. On the $35^{\text {th }}$ day, all mice were sacrificed by $\mathrm{CO}_{2}$ inhalation. $\mathrm{CO}_{2}$ was delivered in a predictable and regulated method at a low flow rate of $10-30 \%$ volume displacement $/ \mathrm{min}$. Animal mortalities were confimed by trained personnel, who recognized the arrest of vital signs in the animals. The tumor tissues were paraffin-embedded for immunohistochemical analysis of Ki-67. Bioluminescent imaging was used to detect intracranial tumor growth on the $35^{\text {th }}$ day. Animal studies were conducted according to the recommendations outlined in the Guide for the Care and Use of Laboratory Animals in the Weatherall report (17). Animal experiments were approved by the Committee on the Ethics of Animal Experiments of Hebei University (Baoding, China).

Breast invasive carcinoma (BRCA) microarray data. The Cancer Genome Atlas (TCGA; http://cancergenome.nih.gov) data were downloaded from the University of California Santa Cruz(UCSC) Cancer Genome Browser (https://genome-cancer. ucsc.edu/proj/site/hgHeatmap/). The Agilent custom arrays (Agilent G4502A_07_3 array; n=597) were used in the study (18). The UCSC Cancer Genome Browser is a suite of web-based tools used for the visualization, integration and analysis of cancer genomics, and associated clinical data.

Cancer Landscapes analysis. Cancer Landscapes is an online-based statistical network model developed by Professor Sven Nelander from Uppsala University (Uppsala, Sweden), which provides high-performance statistical network modeling of multiple human cancer. The network model was constructed using data from Cancer Landscapes (www.cancerlandscapes. org; version beta).

Analysis of differentially expressed genes (DEGs). Total RNA was isolated from the MDA-MB-231 cells using TRIzol ${ }^{\circledR}$ reagent (Invitrogen; Thermo Fisher Scientific, Inc.) according to the manufacturer's protocol. The RNA-Sequecncing data were generated with Illumina sequencing technology (Bejing Genomics Institute, Shenzhen, China), as previously described (19). The DEGs between different groups were identified using Bioconductor edgeR (version 3.12.0) (20). A Student's t-test was performed to identify the DEGs between the Thiostrepton group and control group. $\mathrm{P}<0.01$ and $\mid \log _{2}$ fold changel $>2$ were selected as the cut-off criterion. Hierarchical clustering were performed using DEG expression values through the MultiExperiment Viewer software version 4.9 (http://mev.tm4.org/\#/welcome) (21). DAVID (version 6.7; http://david.ncifcrf.gov/) was used to detect Kyoto Encyclopedia of Genes and Genomes (KEGG; https://www. genome.jp/kegg/) pathways (22). $\mathrm{P}<0.05$ was selected as the cut-off criterion for significantly enriched KEGG pathways.

STRING protein networks tool. To construct a protein-protein interaction (PPI) network, the STRING database (https://string-db.org/) was used with the cut-off criterion of combined score $>0.7$.

Statistical analysis. Statistical analyses were performed using SPSS 17.0 (SPSS, Inc., Chicago, IL, USA). Experiments were performed and repeated three times with similar results. All data are presented as the mean \pm standard deviation. Student's t-test and one-way analysis of variance followed by Dunnett's post hoc test were used to evaluate differences among groups. $\mathrm{P}<0.05$ was considered to indicate a statistically significant difference.

\section{Results}

Position of FOXM1 in a breast cancer gene network. The key role of FOXM1 in breast cancer was identified in a Cancer Landscapes analysis. A network model was constructed using multidimensional perspective data from breast cancer and 

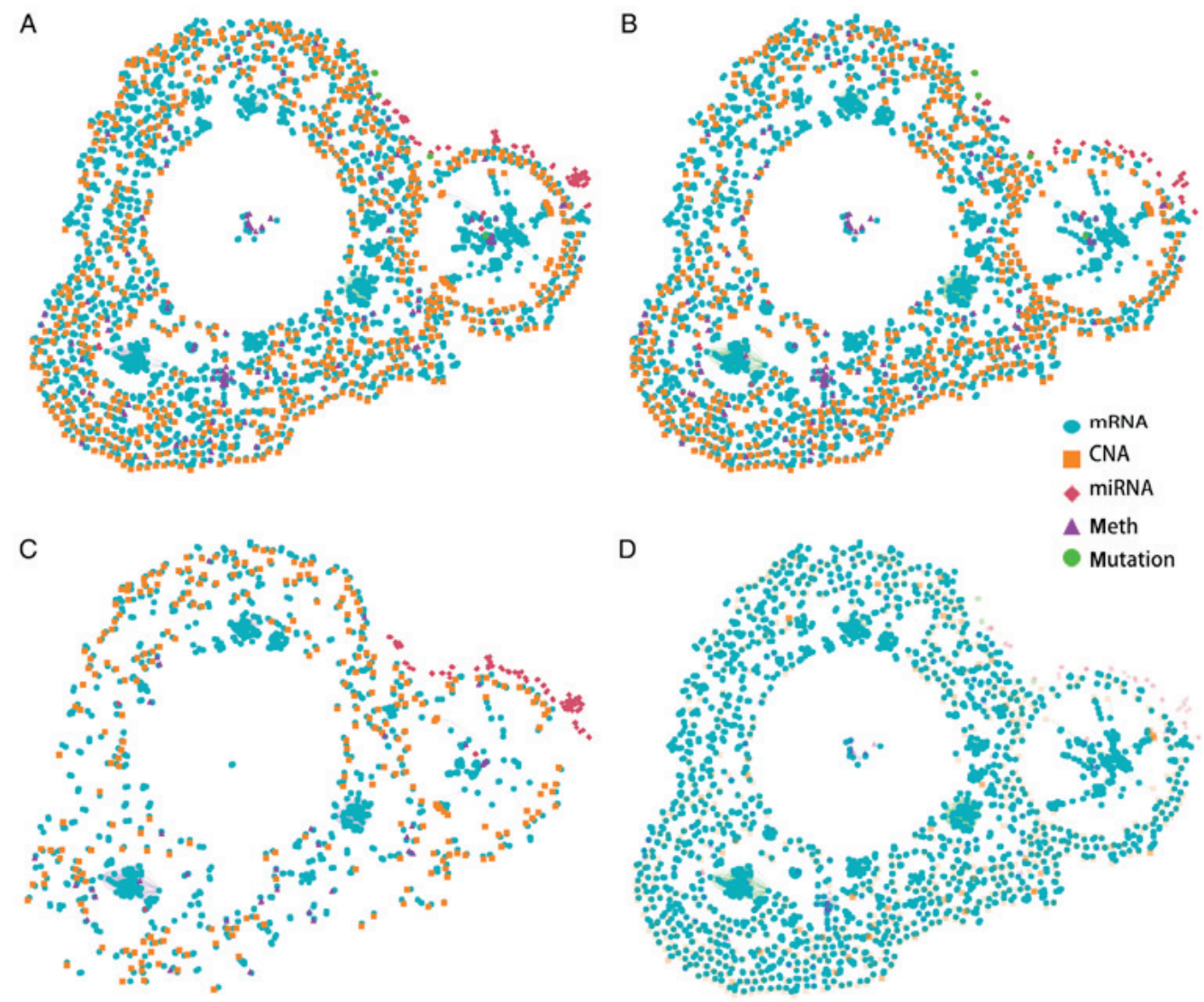

Figure 1. Gene interaction networks for cancer in women. (A) Gene interaction networks in breast and ovarian cancer. (B) Gene interaction network in breast cancer. (C) Gene interaction network in ovarian cancer. (D) mRNA network in breast cancer. CNA, copy number aberration; miRNA, microRNA.

ovarian cancer, which are the two most common tumor types in females. An overview of the gene interaction networks is presented in Fig. 1A, which demonstrated that DNA copy number aberrations (CNAs) and mRNA alterations are characteristic features of these networks. The overlapping genes suggested that the two types of cancer share specific network genes, which suggests that certain gene functions are evolutionarily conserved between the two cancer types. The gene interaction network contains three primary clusters that demonstrate strong similarities in gene expression patterns in the two types of tumors. A previous study demonstrated that basal-like breast cancer and serous ovarian carcinoma are the most similar in terms of genomic mutations and copy number and that these two difficult-to-treat cancer types may share driver mutation events and common therapeutic approaches (18). The present results further suggested that the overlapping genes identified in the network are strongly associated with these tumors. These results suggested that breast and ovarian cancer may have similar molecular pathogenic mechanisms.

The gene networks of the two tissue types were separated (Fig. 1B and C). More mRNA alterations and CNAs were identified in the breast cancer gene network compared with the ovarian cancer gene network. Furthermore, the two types of cancer genes were identified to be tissue-specific. A recent study identified that certain cancer genes are only involved in the development of specific cancer types; however, are rarely identified in other types of cancer (23). This bias is affected by environmental factors and cellular processes. The present study provides a better understanding of the gene interaction networks in the two tissue types.

FOXM1 is a typical proliferation-associated transcription factor, and is essential for cancer initiation and progression (24). To obtain a thorough understanding of the regulation and function of FOXM1 in breast cancer, breast cancer mRNA networks were constructed (Fig. 1D). The results suggested that FOXM1 serves a key role in mRNA networks and in the progression of breast cancer. As FOXM1 is the most important member of the gene network, a further analysis of FOXM1 in breast cancer was conducted.

Expression of FOXM1 and its regulated gene network in breast cancer. FOXM1 was observed to be highly expressed in breast cancer; however, further research was required to determine the different expression patterns and functions of FOXM1 in breast cancer subtypes. To identify the functions and gene networks associated with FOXM1 in breast cancer, a BRCA gene expression dataset from TCGA was used. Using the FOXM1 transcriptional network extracted from the UCSC Cancer Browser website, the expression of FOXM1 in different breast cancer subtypes was analyzed. A gene expression profile analysis revealed that the breast cancer subtypes are associated with the expression of ER, PR and HER2. As presented in Fig. 2A, the expression of FOXM1 is low in the luminal A (ER+/PR+/HER2-) and luminal A/B (ER+/PR+/HER2+/-) subtypes, which are characteristically ER- and PR-positive. In contrast, the HER2+ subtype (ER-/PR-/HER2+) demonstrated slightly increased expression of FOXM1. Notably, it was observed 
A
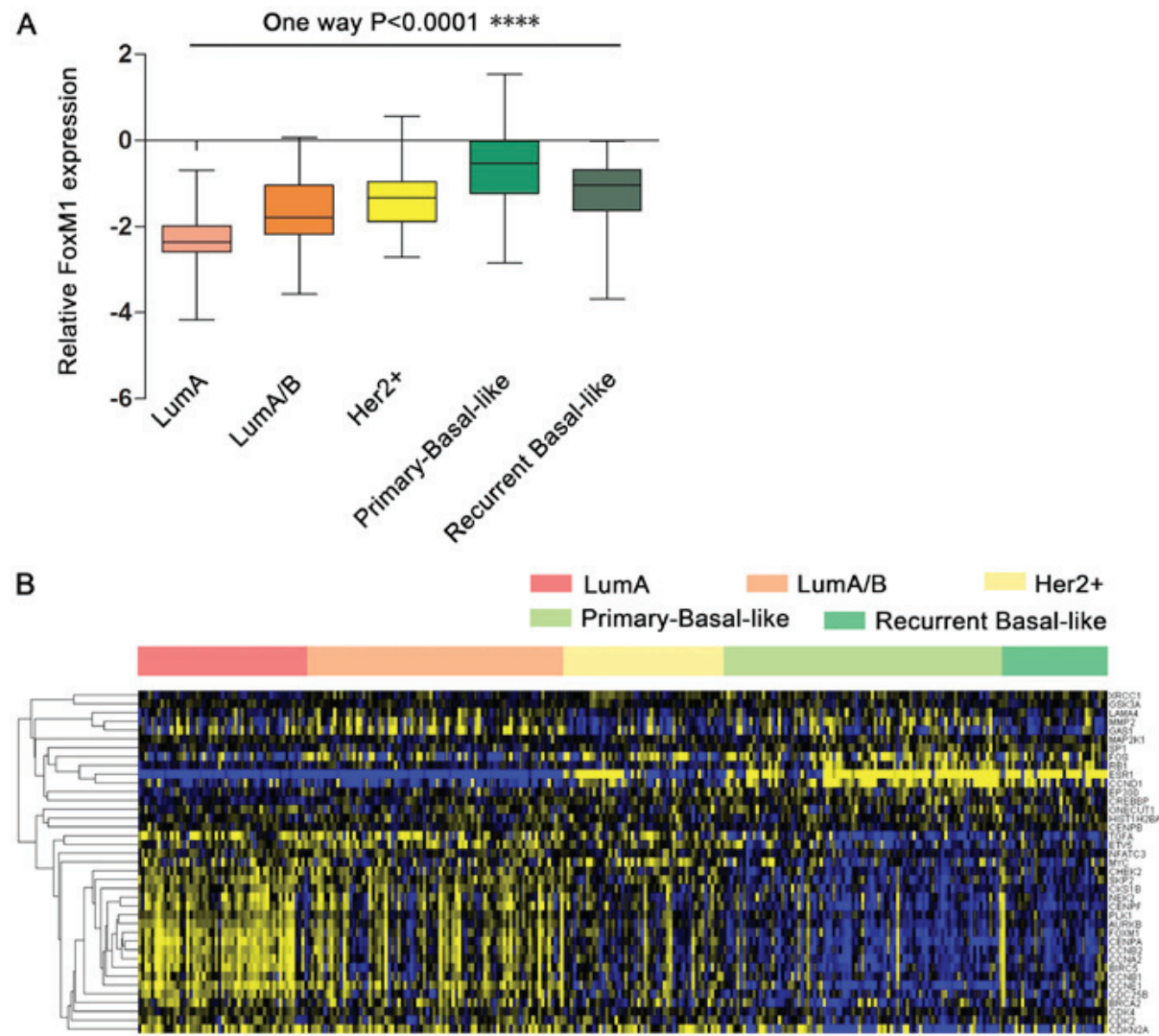

Figure 2. Expression of FOXM1 in clinical samples of breast cancer subtypes. (A) FOXM1 expression was significantly increased in basal-like/TNBC tumors compared with non-basal-like tumors. ${ }^{* * * *} \mathrm{P}<0.0001$. (B) Heatmap representation of the expression of the FOXM1 regulatory network in different breast cancer subtype patient samples. The heatmap presents 38 direct FOXM1-associated genes in breast cancer subtypes. Blue indicates upregulated and yellow indicates downregulated. FOXM1, forkhead box M1; Lum, luminal; HER2, human epidermal growth factor receptor 2.

that primary and recurrent tumors of the basal-like breast cancer subtype (typically characterized by ER-/PR-/HER2status) exhibited stronger expression of FOXM1. Furthermore, FOXM1 expression was slightly decreased in recurrent breast cancer compared with primary breast cancer. These results suggested that the differential FOXM1 expression across the various subtypes of breast cancer is associated with the ER, PR and HER2 status. ER/PR may function with HER2 to contribute to differential FOXM1 expression in breast cancer subtypes. ER-/PR-/HER2- status (characteristic of the basal-like or TNBC subtypes) is more positively associated with FOXM1 expression compared with the other breast cancer subtypes.

To identify the functions of FOXM1 regulatory networks in breast cancer, the expression of FOXM1 and FOXM1-regulated genes was further analyzed. A heatmap demonstrated that 38 genes are directly associated with FOXM1 in breast cancer, including coregulators (RB transcriptional corepressor 1) and target genes (Fig. 2B). The signature of 38 FOXM1-regulated genes downregulated by treatment with Thiostrepton was validated by previously published data (25).

FOXM1 expression was increased in the basal-like breast cancer subtype compared with the other breast cancer subtypes. Furthermore, FOXM1 target genes were upregulated in the basal-like breast cancer subtype and were associated with FOXM1 expression. Genes with expression that was highly associated with FOXM1 expression in basal-like breast cancer were further investigated. Two principal groups of cell cycle-associated genes have been defined; those that demonstrated peak expression in $\mathrm{S}$ phase $\left(\mathrm{G}_{1} / \mathrm{S}\right)$ and those whose expression peaked in mitosis $\left(\mathrm{G}_{2} / \mathrm{M}\right)$ (26). According to the present data, numerous FOXM1-associated genes were expressed in $S$ phase $\left(G_{1} / S\right)$, including $G_{1} / S$-specific cyclin $E-1$, cyclin-dependent kinase 2, M-phase inducer phosphatase 2 (CDC25B), S-phase kinase-associated protein 2, breast cancer type 1 susceptibility protein and breast cancer type 2 susceptibility protein. Other specific genes were critical for $\mathrm{G}_{2}-\mathrm{M}$ progression, including CCNB2, CCNB1, CCNA2, PLK1, aurora kinase B, serine/threonine-protein kinase 2, CHEK2, CDC25B, baculoviral IAP repeat containing protein 5 , centromere protein $\mathrm{A}$, centromere protein $\mathrm{E}$ and centromere protein $\mathrm{F}$. This observation emphasized the role of FOXM1 as a core gene in the enhanced proliferation signature in breast cancer subtypes. Other genes that are known to be associated with cancer, including matrix metalloproteinase 2, are associated with the role of FOXM1 in metastasis. These results demonstrated the core function of FOXM1 within its regulatory network. Increased FOXM1 expression demonstrated good predictive ability for the diagnosis of TNBC.

Differential gene expression profiling in breast cancer cell lines treated with Thiostrepton. To investigate the function of FOXM1 in TNBC in vitro, RNA-sequencing technology was used to analyze RNA expression following treatment of the MDA-MB-231 breast cancer cell line with Thiostrepton. Thiostrepton, a specific inhibitor of 
A

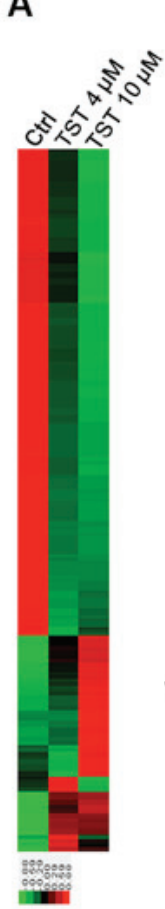

B

Thyroenoid backbone biosynthesis 3

Thyroid hormone signaling pathway
GnRH signaling pathway 6

Neurotrophin signaling pathway

Tryptophan metabolism 4

Small cell lung cancer

ErbB signaling pathway

Pathways in cancer

4

Jak-STAT signaling pathway

Oxytocin signaling pathway

Melanogenesis

4

Metabolic pathways

Pyruvate metabolism

Wnt signaling pathway 5

Amphetamine addiction

Long-term potentiation

ECM-receptor interaction 14

clcium signaling path wos

Propanoate metabolism 13

Phagosome 6

Focal adhesion 12

Notch signaling pathway

P13K-Akt signaling pathway

Glycolysis / Gluconeogenesis

Pentose phosphate pathway

Fatty acid metabolism

Fscherichia coli infection

Gap junction

Endocytosis
${ }_{9}^{8}$

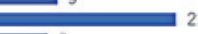

$\operatorname{lin}_{9}^{8} 9$

9

10

12

12 27
C

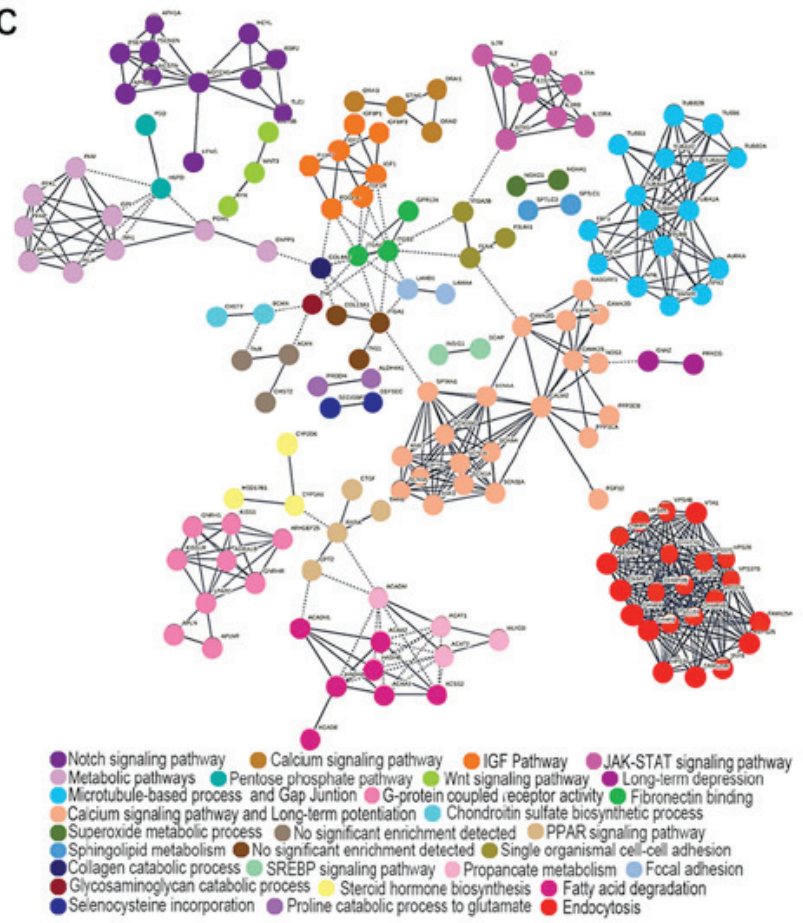

Figure 3. Differential gene expression profiling in MDA-MB-231 cells treated with TST. (A) Heatmap representation of significantly DEGs in MDA-MB-231 cells following treatment with 4 and $10 \mu \mathrm{l} / \mathrm{ml}$ TST. Red indicates downregulation and green indicates upregulation. (B) Enriched canonical pathways were analyzed by Kyoto Encyclopedia of Genes and Genomes pathway analysis. $\mathrm{P}<0.01$. Genes used in the analysis included the 248 downregulated DEGs that were common to the 4 and $10 \mu \mathrm{l} / \mathrm{ml}$ TST treatment groups. (C) Protein-protein interaction network of the 248 downregulated genes. Nodes represent proteins and edges represent connections between them. Nodes are color-coded and grouped into functional clusters, according to their annotation terms. TST, Thiostrepton; DEGs, differentially expressed genes; Ctrl, control.

FOXM1, was previously demonstrated to induce cell death via a decrease in FOXM1 expression in breast cancer cell lines $(27,28)$. MDA-MB-231 cells were treated with $4 \mu \mathrm{M}$ and $10 \mu \mathrm{M}$ Thiostrepton for $48 \mathrm{~h}$. Following treatment, RNA was extracted and RNA-sequencing analysis was performed. In total, 5,888 significantly differentially expressed genes (DEGs) were identified in MDA-MB-231 cells treated with $10 \mu \mathrm{l} / \mathrm{ml}$ Thiostrepton compared with the control group. Among these genes, 4,200 genes were downregulated and 1,688 genes were upregulated (Fig. 3A). A comparative analysis of cells treated with $4 \mu \mathrm{l} / \mathrm{ml}$ Thiostrepton identified 662 DEGs, of which 165 DEGs were upregulated and 497 DEGs were downregulated. These results suggest that treatment with Thiostrepton altered gene expression in MDA-MB-231 cells in a dose-dependent manner. This observation further emphasizes the important role of FOXM1 in the regulation of gene networks in TNBC.

To identify the biologically meaningful pathways affected by a FOXM1 inhibitor, KEGG pathway enrichment analyses were performed. Specifically, the 248 downregulated DEGs that were common to the 4 and $10 \mu \mathrm{M}$ Thiostrepton treatment groups were selected for further analysis. The top 10 significantly enriched pathways included the following: 'Metabolic pathways', 'Endocytosis', 'PI3K-Akt signaling pathway', 'Focal adhesion', 'Pathways in cancer', 'Proteoglycans in cancer', 'Carbon metabolism', 'Calcium signaling pathway', 'Phagosome' and 'Gap junction' (Fig. 3B). Metabolic pathways were considered significantly enriched pathways. FOXM1 serves a key role in metabolic pathways in TNBC. These results suggested that FOXM1 was highly associated with multiple biological processes in the network.
Determination of PPI networks is a useful method for assessing functional associations among genes that exhibit collective mRNA level differential expression in disease $(29,30)$. The observed dysregulation of PPIs suggested an important mechanism of FOXM1 in TNBC. To obtain a global view of the interactions between proteins in Thiostrepton-treated cells, a PPI network was constructed using STRING. STRING provides a critical assessment and integration of protein-protein interactions, including direct (physical) and indirect (functional) associations (31). Among the number of clusters presented in Fig. 3C, known and predicted interactions arose from the majority of proteins in the network. Proteins, including neurogenic locus notch homolog protein 1 (NOTCH-1), insulin-like growth factor 1 (IGF1), cytokine receptor common subunit $\gamma$ (IL2RG), tubulin $\beta$ (TUBB), calmodulin 2 , integrin $\beta 3$, sodium channel protein type 5 subunit $\alpha$ and charged multivesicular body protein were observed in the hub positions (proteins with multiple edges) in the PPI network. NOTCH-1 is a critical regulator of the development of human breast cancer (32). The knockdown of NOTCH-1 is therapeutically effective in ER $\alpha$-negative breast cancer (33). FOXM1 is a downstream target of NOTCH1 signaling (34). IGF1 has significant growth-promoting activity and serves an important role in the development, progression and metastasis of breast cancer (35). The IL2RG protein is required for T-cell proliferation and other activities crucial to the regulation of the immune response (36). TUBB is a principal constituent of microtubules that binds two molecules of guanosine triphosphate, one at an exchangeable site on the $\beta$ chain and the other at a non-exchangeable site 
A
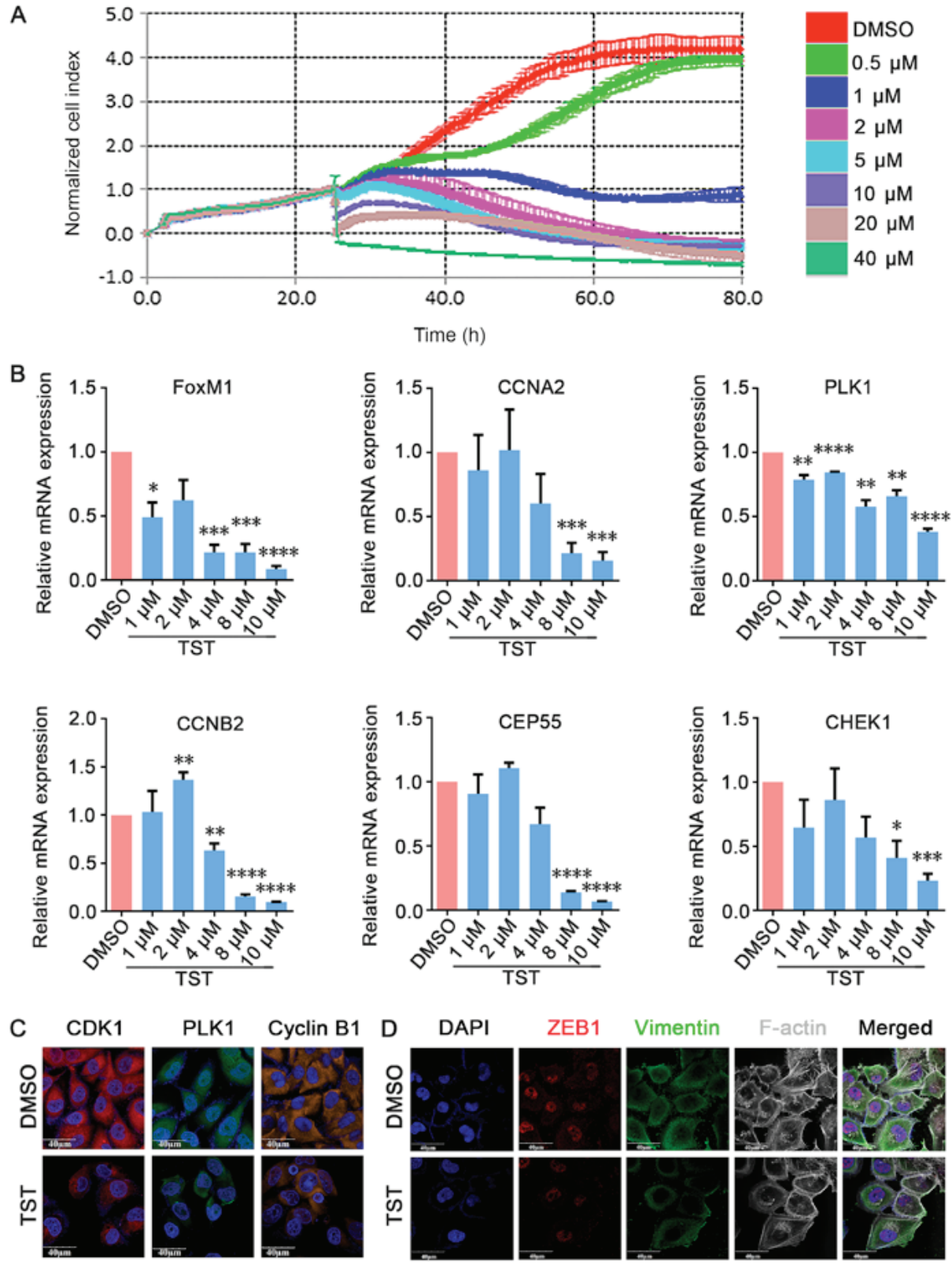

Figure 4. TST inhibits MDA-MB-231 cell growth in vitro. (A) MDA-MB-231 cells were treated with TST at the indicated doses. Cell viability was determined by real-time cell proliferation assay. (B) Cells were treated with $10 \mu \mathrm{mol} / 1 \mathrm{TST}$ for $48 \mathrm{~h}$. The mRNA expression levels of FOXM1, CCNA2, PLK1, CHEK1, CEP55 and CCNB2 were determined by quantitative polymerase chain reaction. (C) Immunofluorescence staining demonstrated that CDK1, PLK1 and cyclin B1 expression decreased upon treatment. (D) Triple immunofluorescence staining demonstrated that ZEB1 co-localized with vimentin and F-actin, and decreased upon treatment. ${ }^{*} \mathrm{P}<0.05,{ }^{* *} \mathrm{P}<0.01,{ }^{* * *} \mathrm{P}<0.001,{ }^{* * * * *} \mathrm{P}<0.0001$ vs. respective DMSO. TST, Thiostrepton; DMSO, dimethyl sulfoxide; FOXM1, forkhead box M1; CCNA2, cyclin A2; PLK1, polo like kinase 1; CHEK1, checkpoint kinase 1; CEP55, centrosomal protein 55; CCNB2, cyclin B2; ZEB1, zinc finger E-box-binding homeobox 1; F-actin, filamentous actin; CDK1, cyclin-dependent kinase 1.

on the $\alpha$ chain (37). FOXM1 is essential for the migration of mesenchymal cells and directly induces integrin- $\beta 3$ expression (38). These results emphasized the multiple important functions of FOXM1 in breast cancer progression at the proteome level.

In summary, through the comprehensive analysis of global pathway regulation in TNBC in response to Thiostrepton, the underlying mechanisms of FOXM1 in the network were demonstrated.

Thiostrepton inhibits TNBC cell growth in vitro. To investigate the effects of Thiostrepton on TNBC cell growth in vitro, MDA-MB-231 cells were treated with increasing concentrations of Thiostrepton and cell proliferation was measured via RTCA. MDA-MB-231 cells were used in the present study and were treated with Thiostrepton for $80 \mathrm{~h}$ at concentrations that ranged between 1 and $40 \mu \mathrm{M}$. The RTCA results demonstrated that the proliferation of MDA-MB-231 cells was suppressed in a dose-dependent manner compared with the negative control group (Fig. 4A). qPCR analysis demonstrated that FOXM1 mRNA expression levels decreased in a dose-dependent manner in Thiostrepton-treated MDA-MB-231 cells (Fig. 4B). To examine the role of FOXM1 and its possible target genes in cell proliferation, qPCR was used to detect the expression of cell cycle-associated genes, including CCNA2, PLK1, CCNB2, CEP55 and CHEK1. As presented in Fig. 4B, it 

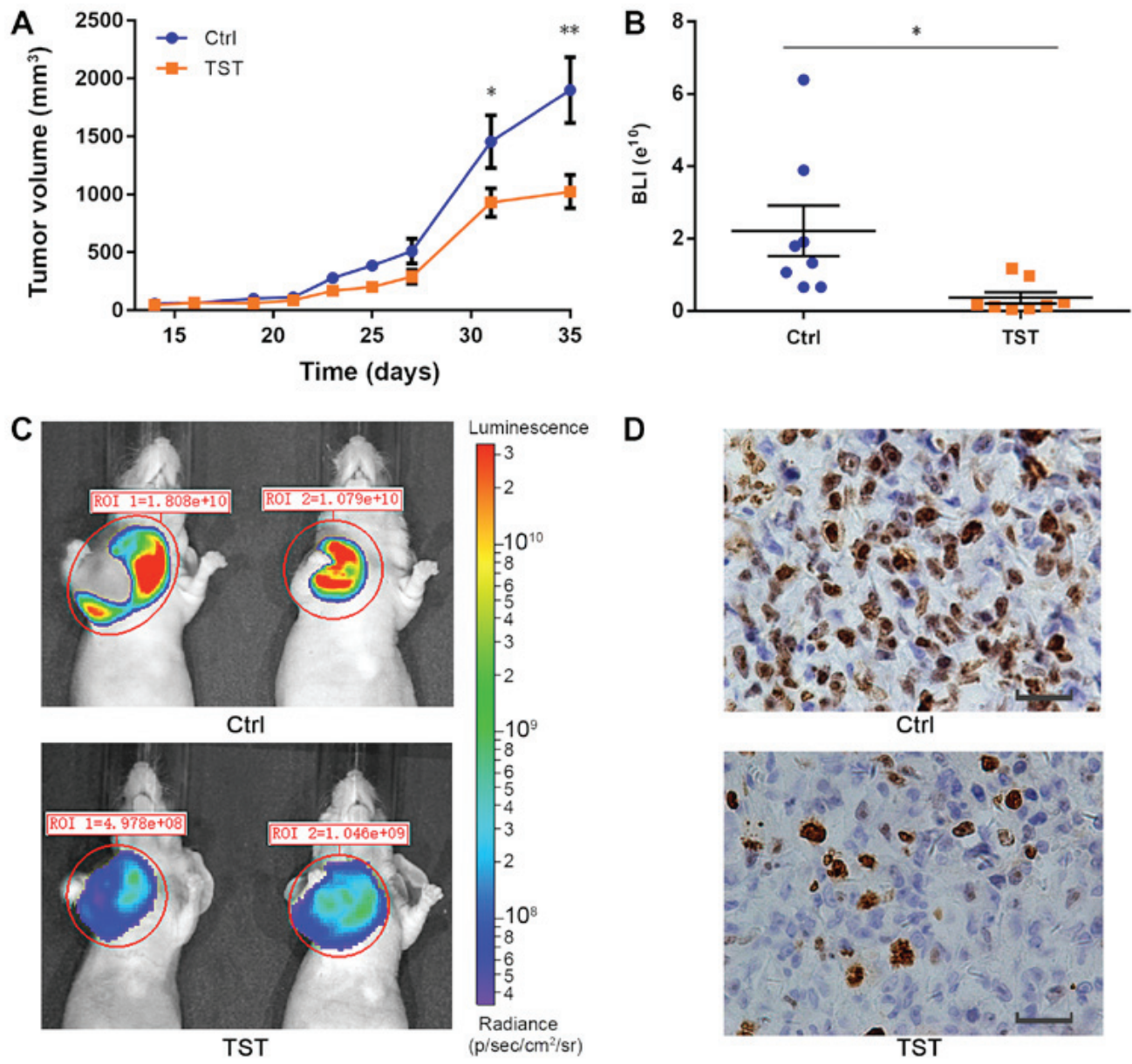

Figure 5. TST suppresses the proliferation of MDA-MB-231 xenografts in vivo. (A) Tumor volumes were estimated in MDA-MB-231 xenografts. $\mathrm{n}=8$. (B) Quantification of BLI signal intensity demonstrated a significant decrease in animals treated with Thiostrepton (orange spot; $\mathrm{n}=8$ ) compared with the control animals (blue spot; $n=8$ ). (C) Representative BLI images of untreated and TST-treated MDA-MB-231 xenografts on day 35. Colors in the BLI images correspond with signal strength/intensity: red, strong; cyan, intermediate; and blue, weak. Colored scale bar represents photons $/ \mathrm{sec} / \mathrm{cm}^{2} / \mathrm{steradian}$. $\mathrm{P}<0.05$. (D) Immunohistochemistry for proliferation marker Ki-67 in tumor sections. Scale bar, $100 \mu \mathrm{m} .{ }^{*} \mathrm{P}<0.05,{ }^{* *} \mathrm{P}<0.01$. TST, Thiostrepton; BLI, bioluminescent; ROI, region of interest; Ctrl, control.

was observed that the expression of these genes decreased in a dose-dependent manner in Thiostrepton-treated MDA-MB-231 cells from $4 \mu \mathrm{M}$ Thiostrepton. These results suggested that Thiostrepton exerted an anti-tumor effect in TNBC cells. Immunofluorescence staining additionally demonstrated that CDK1, PLK1 and CCNB1 expression decreased upon treatment with $10 \mu \mathrm{M}$ Thiostrepton for $48 \mathrm{~h}$ (Fig. 4C). These results suggested that Thiostrepton inhibited cell proliferation by suppressing the expression of cell cycle-associated factors at the mRNA and protein expression levels. These results demonstrated that FOXM1 is functionally essential for the proliferation of TNBC cells in vitro.

FOXM1 serves an important role in the regulation of the epithelial-mesenchymal transition (EMT). EMT is an important feature of TNBC, and a previous study demonstrated that FOXM1 enhances EMT in breast cancer cells (39). Treatment with Panepoxydone, a nuclear factor- $\mathrm{kB}$ inhibitor, downregulated FOXM1 and resulted in a reversal of EMT (40). However, the molecular mechanisms of FOXM1 in EMT and the establishment of distant metastasis remain unclear. To analyze the inhibitory effect of treatment with Thiostrepton on FOXM1-driven EMT, triple immunofluorescence staining of EMT-associated factors (vimentin, F-actin and ZEB1) was performed following treatment with $10 \mu \mathrm{M}$ Thiostrepton for $48 \mathrm{~h}$. ZEB1 influences EMT in breast cancer cells by inhibiting E-cadherin repressors (41). Vimentin is a mesenchymal marker that is expressed during EMT (42). Rearrangement of the F-actin cytoskeleton is a crucial event during EMT (43). ZEB1, vimentin and F-actin co-localized and were decreased in MDA-MB-231 cells upon treatment with Thiostrepton (Fig. 4D). These results suggested that Thiostrepton may inhibit EMT in TNBC cells in vitro and that inhibition of FOXM1 may induce EMT reversal.

Thiostrepton reduces tumorigenesis in TNBC in vivo. A number of previous studies demonstrated that FOXM1 is involved in tumorigenesis and promotes cell proliferation by targeting downstream genes $(44,45)$. A recent study demonstrated that FOXM1 overexpression was correlated with larger tumor size, lymph node metastasis, advanced tumor stage and lymph-vascular invasion (46). To investigate the role of FOXM1 in TNBC tumorigenesis, MDA-MB-231 breast cancer cell xenografts were established in mice. The tumor volume in each animal at the defined time points was measured during the procedure. At day 35 , the average tumor volume in the control mice group was $1,900.8 \pm 801.7 \mathrm{~mm}^{3}$, whereas the tumor volume in the treated group was $1,023.4 \pm 410.6 \mathrm{~mm}^{3}$. 
The average tumor volume was significantly decreased in the treated group compared with the control group $(\mathrm{P}<0.01$; Fig. 5A). Thiostrepton suppressed tumor growth in vivo (Fig. 5A-C). Furthermore, using immunohistochemistry, the expression of $\mathrm{Ki}-67$, a protein associated with cell proliferation, was detected. The expression of Ki-67 was decreased following treatment with Thiostrepton compared with the control treatment (Fig. 5D). These results suggested that although Thiostrepton may inhibit tumor proliferation, it was not able to completely inhibit tumor growth in vivo. Other mechanisms may contribute to the tumorigenesis of TNBC. These results provide a better understanding of the important role served by FOXM1 in TNBC tumorigenesis. In the present study, the formation of one small tumor was observed in the liver of a mouse in the control group (data not shown). No distant metastases were observed in the treatment group. Therefore, Thiostrepton slightly inhibits the metastasis of TNBC tumors in vivo.

\section{Discussion}

In the present study, it was identified that FOXM1 serves a central role in breast cancer gene networks, which provide a rich resource for the study of the molecular mechanisms of FOXM1. Using an mRNA network, FOXM1 was identified as the most important member of the gene network. Further experiments were used to validate this hypothesis. FOXM1 was examined at the gene expression level to better elucidate the molecular mechanisms of FOXM1 in a complex gene interaction network. These results suggested that targeting FOXM1 or its network members may be a potential therapeutic strategy for TNBC.

FOXM1 is overexpressed in breast cancer, including TNBC $(47,48)$. Although multiple molecular characteristics of TNBC have been previously identified, the molecular mechanisms of FOXM1 in TNBC have not been fully elucidated (49). Breast cancer is a heterogeneous disease. Different breast cancer subtypes exhibit different histopathological features, biological features, treatment responses and prognoses (50). In the present study, FOXM1 expression patterns were examined in different subtypes of breast cancer. FOXM1 and its associated regulatory network was most markedly expressed in the TNBC subtype compared with other breast cancer subtypes. This provides a better understanding of the highly aggressive nature of TNBC. It was additionally observed that FOXM1 expression was slightly decreased in recurrent breast cancer compared with primary breast cancer. This genetic disparity becomes highly relevant when the application of targeted molecular therapies for primary and recurrent tumors is considered (51). Genomic discordance may result in differences in therapeutic response. The present study suggested that FOXM1 may be a potential therapeutic target for primary breast tumors and recurrent breast cancer. Patients with primary breast tumors may experience more therapeutic benefits compared with patients with recurrent tumors. However, additional studies are required to validate this.

The present study examined the role of FOXM1 and aimed to map global gene networks in TNBC cell lines, and to clarify their association with the biological function of FOXM1. It was identified that FOXM1 regulates certain cell cycle genes and affects TNBC proliferation, which is consistent with previous studies $(52,53)$. The pathway analysis demonstrated that FOXM1 is highly associated with multiple biological processes in the network. The present study confirmed previous results and further established that FOXM1 has numerous functions in TNBC in addition to cell cycle regulation. FOXM1 regulates a generalized gene network. 'Metabolic pathways', 'Endocytosis' and 'PI3K-Akt signaling pathway' were the top three enriched pathways following treatment with Thiostrepton. There are a number of different aspects of the inhibitory effects of Thiostrepton. Metabolic pathways were considerably enriched among the pathways. Notably, tumor metabolism is associated with the tumor microenvironment and tumor progression (54). Recently, it was demonstrated that FOXM1 expression is associated with glucose metabolism in cancer cells (55). FOXM1 promotes the reprogramming of glucose metabolism in human hepatocellular carcinoma cells and is considered a novel transcriptional regulator of glycolysis (55). The present results suggested that treatment with Thiostrepton may downregulate metabolic processes in MDA-MB-231 cells. It was additionally identified that IGF1 and its interaction network are consistent with the pathway enrichment analysis. IGF1 has high growth-promoting activity. IGF1 has an important role in breast cancer development, progression and metastasis (56). The present study suggested that FOXM1 serves an important role in the regulation of metabolism during TNBC progression. Further studies are required to elucidate the mechanisms underlying this regulation.

Thiostrepton, a natural product originally isolated from Streptomyces azureus, has attracted increasing attention in the field of breast cancer therapy due to its potential anti-cancer activity as a FOXM1 inhibitor (57). In the present study, the FOXM1 gene was investigated; MDA-MB-231 cells were treated with 4 and $10 \mu \mathrm{l} / \mathrm{ml}$ Thiostrepton, and the expression profiles were subsequently analyzed by RNA-sequencing. KEGG and STRING analyses provided a clear description of DEGs following treatment with Thiostrepton. The variety of pathways suggested an extensive impact of Thiostrepton on breast cancer cells. It is crucial to identify the specific mechanism of FOXM1, and in the present study, the function of FOXM1 in the cell cycle was identified. The FOXM1-associated genes were defined by the UCSC Cancer Browser. All these genes were associated with the cell cycle, and a number of them were inhibited by treatment with Thiostrepton, which was confirmed by real-time PCR and RNA-sequencing. Taken together, these data suggested that FOXM1 was able to influence multiple aspects of breast cancer cells and that cell cycle-associated pathways are a key mechanism affected by FOXM1. In the present study, Thiostrepton exhibited anti-cancer activity in TNBC in vivo and in vitro. Although Thiostrepton may inhibit tumor proliferation, it is not able to completely inhibit tumor growth and metastasis in vivo. Therefore, there may be other mechanisms that contribute to the tumorigenesis of TNBC. One of the leading causes of resistance to small molecule inhibitors is cross-talk between dysregulated survival pathways (58). Recent studies demonstrated that targeting multiple components of different pathways with a combination of specific inhibitors is more effective 
compared with treatment with a single agent alone $(59,60)$. A previous study observed that the combined targeting of cyclooxygenase- 2 and FOXM1 causes inhibition of invasion and migration, reduction in cell viability and induction of apoptosis in colorectal cancer cells (61). Due to the complicated molecular mechanisms of FOXM1 in TNBC, a combined therapy using multiple targeting agents for TNBC treatment is highly recommended. This may provide more effective therapeutic strategies for TNBC.

In conclusion, the present study provides a better understanding of the molecular mechanisms of FOXM1 within a complex gene interaction network. FOXM1 serves a critical role in the regulatory network in TNBC. FOXM1 may be a promising molecular therapeutic target for TNBC.

\section{Acknowledgements}

Not applicable.

\section{Funding}

The present study was supported by the Hebei Provincial Top Level Talents Funded Projects of China (grant no. CY201601) and the Hebei Province Technical Innovation Guidance Funded Projects of China (grant no. 18247792D).

\section{Availability of data and materials}

The datasets used and/or analyzed during the study are available from the corresponding author on reasonable request.

\section{Authors' contributions}

$\mathrm{BZ}$ and YT conceived and designed the experiments. YT was responsible for writing the manuscript. QW conducted the bioinformatics analyses. YX, XQ, SZ, YW and YY performed the experiments and analyzed the data. All authors read and approved the final version of the manuscript.

\section{Ethics approval and consent to participate}

Animal studies were conducted according to the recommendations outlined in the Guide for the Care and Use of Laboratory Animals in the Weatherall report. Animal experiments were approved by the Committee on the Ethics of Animal Experiments of Hebei University (Baoding, China).

\section{Patient consent for publication}

Not applicable.

\section{Competing interests}

The authors declare that they have no competing interests.

\section{References}

1. Torre LA, Bray F, Siegel RL, Ferlay J, Lortet-Tieulent J and Jemal A: Global cancer statistics, 2012. CA Cancer J Clin 65: $87-108,2015$
2. Khongkow P, Gomes AR, Gong C, Man EP, Tsang JW, Zhao F Monteiro LJ, Coombes RC, Medema RH, Khoo US, et al: Paclitaxel targets FOXM1 to regulate KIF20A in mitotic catastrophe and breast cancer paclitaxel resistance. Oncogene 35: 990-1002, 2016.

3. Borin TF, Angara K, Rashid MH, Achyut BR and Arbab AS: Arachidonic acid metabolite as a novel therapeutic target in breast cancer metastasis. Int J Mol Sci 18: pii: E2661, 2017.

4. Arnold KM, Pohlig RT and Sims-Mourtada J: Co-activation of Hedgehog and Wnt signaling pathways is associated with poor outcomes in triple negative breast cancer. Oncol Lett 14: 5285-5292, 2017.

5. McGuire A, Lowery AJ, Kell MR, Kerin MJ and Sweeney KJ: Locoregional recurrence following breast cancer surgery in the trastuzumab era: A systematic review by subtype. Ann Surg Oncol 24: 3124-3132, 2017.

6. Gu G, Dustin D and Fuqua SA: Targeted therapy for breast cancer and molecular mechanisms of resistance to treatment. Curr Opin Pharmacol 31: 97-103, 2016.

7. Liu J, Xiao Y, Wei W, Guo JX, Liu YC, Huang XH, Zhang RX, Wu YJ and Zhou J: Clinical efficacy of administering oxaliplatin combined with S-1 in the treatment of advanced triple-negative breast cancer. Exp Ther Med 10: 379-385, 2015.

8. Reaz S, Tamkus D and Andrechek ER: Using gene expression data to direct breast cancer therapy: Evidence from a preclinical trial. J Mol Med (Berl) 96: 111-117, 2018.

9. Guo GC, Wang JX, Han ML, Zhang LP and Li L: microRNA-761 induces aggressive phenotypes in triple-negative breast cancer cells by repressing TRIM29 expression. Cell Oncol (Dordr) 40: 157-166, 2017.

10. Lam EW and Gomes AR: Forkhead box transcription factors in cancer initiation, progression and chemotherapeutic drug response. Front Oncol 4: 305, 2014.

11. Abdeljaoued S, Bettaieb I, Nasri M, Adouni O, Goucha A, El Amine O, Boussen H, Rahal K and Gamoudi A: Overexpression of FOXM1 Is a Potential Prognostic Marker in Male Breast Cancer. Oncol Res Treat 40: 167-172, 2017.

12. Lam AK, Ngan AW, Leung MH, Kwok DC, Liu VW, Chan DW, Leung WY and Yao KM: FOXM1b, which is present at elevated levels in cancer cells, has a greater transforming potential than FOXM1c. Front Oncol 3: 11, 2013.

13. Hamurcu Z, Kahraman N, Ashour A and Ozpolat B: FOXM1 transcriptionally regulates expression of integrin $\beta 1$ in triple-negative breast cancer. Breast Cancer Res Treat 163: 485-493, 2017.

14. Lee JJ, Lee HJ, Son BH, Kim SB, Ahn JH, Ahn SD, Cho EY and Gong G: Expression of FOXM1 and related proteins in breast cancer molecular subtypes. Int J Exp Pathol 97: 170-177, 2016.

15. Hamurcu Z, Ashour A, Kahraman N and Ozpolat B: FOXM1 regulates expression of eukaryotic elongation factor 2 kinase and promotes proliferation, invasion and tumorgenesis of human triple negative breast cancer cells. Oncotarget 7: 16619-16635, 2016.

16. Livak KJ and Schmittgen TD: Analysis of relative gene expression data using real-time quantitative PCR and the 2(-Delta Delta C(T)) Method. Methods 25: 402-408, 2001.

17. National Research Council (US) Institute for Laboratory Animal Research: Guide for the Care and Use of Laboratory Animals. National Academies Press (US), Washington, DC, 1996.

18. Cancer Genome Atlas Network: Comprehensive molecular portraits of human breast tumours. Nature 490: 61-70, 2012.

19. Xu Y, Li W, Liu X, Ma H, Tu Z and Dai Y: Analysis of microRNA expression profile by small RNA sequencing in Down syndrome fetuses. Int J Mol Med 32: 1115-1125, 2013.

20. Yang M, Li H, Li Y, Ruan Y and Quan C: Identification of genes and pathways associated with MDR in MCF-7/MDR breast cancer cells by RNA-seq analysis. Mol Med Rep 17: 6211-6226, 2018.

21. Eisen MB, Spellman PT, Brown PO and Botstein D: Cluster analysis and display of genome-wide expression patterns. Proc Natl Acad Sci USA 95: 14863-14868, 1998.

22. Liu C, Chen N, Huang K, Jiang M, Liang H, Sun Z, Tian J and Wang D: Identifying hub genes and potential mechanisms associated with senescence in human annulus cells by gene expression profiling and bioinformatics analysis. Mol Med Rep 17: 3465-3472, 2018.

23. Polak P, Karlić R, Koren A, Thurman R, Sandstrom R, Lawrence M, Reynolds A, Rynes E, Vlahoviček K, Stamatoyannopoulos JA, et al: Cell-of-origin chromatin organization shapes the mutational landscape of cancer. Nature 518: 360-364, 2015. 
24. Zona S, Bella L, Burton MJ, Nestal de Moraes G and Lam EW: FOXM1: An emerging master regulator of DNA damage response and genotoxic agent resistance. Biochim Biophys Acta 1839: 1316-1322, 2014

25. Sanders DA, Ross-Innes CS, Beraldi D, Carroll JS and Balasubramanian S: Genome-wide mapping of FOXM1 binding reveals co-binding with estrogen receptor alpha in breast cancer cells. Genome Biol 14: R6, 2013.

26. Fischer $M$ and Müller GA: Cell cycle transcription control DREAM/MuvB and RB-E2F complexes. Crit Rev Biochem Mol Biol 52: 638-662, 2017.

27. Kwok JMM, Myatt SS, Marson CM, Coombes RC, Constantinidou D and Lam EW: Thiostrepton selectively targets breast cancer cells through inhibition of forkhead box M1 expression. Mol Cancer Ther 7: 2022-2032, 2008.

28. Yang N, Zhou TC, Lei XX, Wang C, Yan M, Wang ZF, Liu W, Wang J, Ming KH, Wang BC, et al: Inhibition of Sonic Hedgehog Signaling Pathway by Thiazole Antibiotic Thiostrepton Attenuates the CD44+/CD24-Stem-Like Population and Sphere-Forming Capacity in Triple-Negative Breast Cancer. Cell Physiol Biochem 38: 1157-1170, 2016.

29. Chuang HY, Lee E, Liu YT, Lee D and Ideker T: Network-based classification of breast cancer metastasis. Mol Syst Biol 3: 140, 2007.

30. Chowdhury SA, Nibbe RK, Chance MR and Koyutürk M: Subnetwork state functions define dysregulated subnetworks in cancer. J Comput Biol 18: 263-281, 2011.

31. Szklarczyk D, Franceschini A, Wyder S, Forslund K, Heller D, Huerta-Cepas J, Simonovic M, Roth A, Santos A, Tsafou KP, et al: STRING v10: Protein-protein interaction networks, integrated over the tree of life. Nucleic Acids Res 43 (D1): D447-D452, 2015.

32. Li L, Zhao F, Lu J, Li T, Yang H, Wu C and Liu Y: Notch-1 signaling promotes the malignant features of human breast cancer through NF-кB activation. PLoS One 9: e95912, 2014

33. Rizzo P, Miao H, D'Souza G, Osipo C, Song LL, Yun J, Zhao H, Mascarenhas J, Wyatt D, Antico G, et al: Cross-talk between notch and the estrogen receptor in breast cancer suggests novel therapeutic approaches. Cancer Res 68: 5226-5235, 2008.

34. Wang Z, Li Y, Ahmad A, Banerjee S, Azmi AS, Kong D, Wojewoda C, Miele L and Sarkar FH: Down-regulation of Notch-1 is associated with Akt and FoxM1 in inducing cell growth inhibition and apoptosis in prostate cancer cells. J Cell Biochem 112: 78-88, 2011.

35. Christopoulos PF, Msaouel P and Koutsilieris M: The role of the insulin-like growth factor-1 system in breast cancer. Mol Cancer 14: 43, 2015

36. Suzuki S, Iwamoto M, Saito Y, Fuchimoto D, Sembon S, Suzuki M, Mikawa S, Hashimoto M, Aoki Y, Najima Y, et al: Il2rg gene-targeted severe combined immunodeficiency pigs. Cell Stem Cell 10: 753-758, 2012.

37. Kopp S, Slumstrup L, Corydon TJ, Sahana J, Aleshcheva G, Islam T, Magnusson NE, Wehland M, Bauer J, Infanger M, et al: Identifications of novel mechanisms in breast cancer cells involving duct-like multicellular spheroid formation after exposure to the Random Positioning Machine. Sci Rep 6: 26887, 2016.

38. Malin D, Kim IM, Boetticher E, Kalin TV, Ramakrishna S, Meliton L, Ustiyan V, Zhu X and Kalinichenko VV: Forkhead box F1 is essential for migration of mesenchymal cells and directly induces integrin-beta3 expression. Mol Cell Biol 27: 2486-2498, 2007.

39. Xue J, Lin X, Chiu WT, Chen YH, Yu G, Liu M, Feng XH, Sawaya R, Medema RH, Hung MC, et al: Sustained activation of SMAD3/SMAD4 by FOXM1 promotes TGF- $\beta$-dependent cancer metastasis. J Clin Invest 124: 564-579, 2014.

40. Arora R, Yates C, Gary BD, McClellan S, Tan M, Xi Y, Reed E, Piazza GA, Owen LB and Dean-Colomb W: Panepoxydone targets NF-kB and FOXM1 to inhibit proliferation, induce apoptosis and reverse epithelial to mesenchymal transition in breast cancer. PLoS One 9: e98370, 2014.

41. LiX, Roslan S,Johnstone CN, Wright JA,Bracken CP, Anderson M, Bert AG, Selth LA, Anderson RL, Goodall GJ, et al: MiR-200 can repress breast cancer metastasis through ZEB1-independent but moesin-dependent pathways. Oncogene 33: 4077-4088, 2014.
42. Park MY, Kim KR, Park HS, Park BH, Choi HN, Jang KY, Chung MJ, Kang MJ, Lee DG and Moon WS: Expression of the serum response factor in hepatocellular carcinoma: Implications for epithelial-mesenchymal transition. Int J Oncol 31: 1309-1315, 2007.

43. Shankar J and Nabi IR: Correction: Actin cytoskeleton regulation of epithelial mesenchymal transition in metastatic cancer cells. PLoS One 10: e0132759, 2015.

44. Yang C, Chen H, Tan G, Gao W, Cheng L, Jiang X, Yu L and Tan Y: FOXM1 promotes the epithelial to mesenchymal transition by stimulating the transcription of Slug in human breast cancer. Cancer Lett 340: 104-112, 2013.

45. Halasi M and Gartel AL: Targeting FOXM1 in cancer. Biochem Pharmacol 85: 644-652, 2013.

46. Song X, Fiati Kenston SS, Zhao J, Yang D and Gu Y: Roles of FoxM1 in cell regulation and breast cancer targeting therapy. Med Oncol 34: 41, 2017.

47. Ahn H, Sim J, Abdul R, Chung MS, Paik SS, Oh YH, Park CK and Jang $\mathrm{K}$ : Increased expression of forkhead box M1 is associated with aggressive phenotype and poor prognosis in estrogen receptor-positive breast cancer. J Korean Med Sci 30: 390-397, 2015.

48. Wierstra I: FOXM1 (Forkhead box M1) in tumorigenesis: Overexpression in human cancer, implication in tumorigenesis, oncogenic functions, tumor-suppressive properties, and target of anticancer therapy. Adv Cancer Res 119: 191-419, 2013.

49. Bayraktar R, Ivan C, Bayraktar E, Kanlikilicer P, Kabil NN, Kahraman N, Mokhlis HA, Karakas D, Rodriguez-Aguayo C, Arslan A, et al: Dual Suppressive Effect of miR-34a on the FOXM1/eEF2-Kinase Axis Regulates Triple-Negative Breast Cancer Growth and Invasion. Clin Cancer Res 24: 4225-4241, 2018.

50. Yersal O and Barutca S: Biological subtypes of breast cancer: Prognostic and therapeutic implications. World J Clin Oncol 5: 412-424, 2014

51. Krøigård $A B$, Larsen $M J$, Thomassen $M$ and Kruse TA: Molecular concordance between primary breast cancer and matched metastases. Breast J 22: 420-430, 2016.

52. Wierstra I and Alves J: FOXM1, a typical proliferation-associated transcription factor. Biol Chem 388: 1257-1274, 2007.

53. Lv C, Zhao G, Sun X, Wang P, Xie N, Luo J and Tong T: Acetylation of FOXM1 is essential for its transactivation and tumor growth stimulation. Oncotarget 7: 60366-60382, 2016.

54. Kroemer G and Pouyssegur J: Tumor cell metabolism: Cancer's Achilles' heel. Cancer Cell 13: 472-482, 2008.

55. Shang R, Pu M, Li Y and Wang D: FOXM1 regulates glycolysis in hepatocellular carcinoma by transactivating glucose transporter 1 expression. Oncol Rep 37: 2261-2269, 2017.

56. Lei $\mathrm{T}$ and Ling $\mathrm{X}$ : IGF-1 promotes the growth and metastasis of hepatocellular carcinoma via the inhibition of proteasome-mediated cathepsin B degradation. World J Gastroenterol 21: 10137-10149, 2015.

57. Hegde NS, Sanders DA, Rodriguez R and Balasubramanian S: The transcription factor FOXM1 is a cellular target of the natural product thiostrepton. Nat Chem 3: 725-731, 2011.

58. Rahman MA, Amin AR and Shin DM: Chemopreventive potential of natural compounds in head and neck cancer. Nutr Cancer 62: 973-987, 2010.

59. Tolcher AW, Peng W and Calvo E: Rational Approaches for Combination Therapy Strategies Targeting the MAP Kinase Pathway in Solid Tumors. Mol Cancer Ther 17: 3-16, 2018.

60. Yang N, Wang C, Wang Z, Zona S, Lin SX, Wang X, Yan M, Zheng FM, Li SS, Xu B, et al: FOXM1 recruits nuclear Aurora kinase A to participate in a positive feedback loop essential for the self-renewal of breast cancer stem cells. Oncogene 36: 3428-3440, 2017.

61. Ahmed M, Hussain AR, Siraj AK, Uddin S, Al-Sanea N, Al-Dayel F, Al-Assiri M, Beg S and Al-Kuraya KS: Co-targeting of Cyclooxygenase-2 and FoxM1 is a viable strategy in inducing anticancer effects in colorectal cancer cells. Mol Cancer 14: 131, 2015.

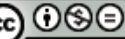

This work is licensed under a Creative Commons Attribution-NonCommercial-NoDerivatives 4.0 International (CC BY-NC-ND 4.0) License. 